\title{
Terapéutica de apoyo en la mucormicosis
}

\section{Therapeutic support in mucormycosis}

\author{
Rodrigo Iñiguez $C^{1}$, Jimena Cevo $E^{2}$, Ximena Fonseca $A^{1}$.
}

\begin{abstract}
RESUMEN
La mucormicosis es una infección infrecuente, causada por hongos oportunistas, pertenecientes al orden de los mucorales, cuyo sello es la invasión vascular por hifas, que conduce a la trombosis arterial. Generalmente afecta a pacientes con una enfermedad de base y produce una infección invasiva, frecuentemente fulminante.

A continuación analizamos el caso de un paciente de 13 años con leucemia linfática aguda y neutropenia secundaria a la quimioterapia, cuyo cuadro clínico se presentó con cefalea fiebre y rinorrea. Las imágenes evidenciaron un compromiso de la pared lateral del seno esfenoidal. Fue tratado con Anfotericina B liposomal, factor de crecimiento de neutrófilos y resección quirúrgica en dos oportunidades con buena respuesta clínica y radiológica.
\end{abstract}

Palabras clave: mucormicosis, anfotericina B liposomal, factor de crecimiento de neutrófilos.

\section{SUMMARY}

Mucormycosis is an infrequent infection, caused by opportunistic fungi, that belong to the order of the mucorals, whose seal is vascular invasion through hypha, and that leads to arterial thrombosis. It generally affects patients with a basic disease and produces an invasive infection, which is frequently fulminating.

We will now analyze the case of a 13 year old patient suffering from acute lymphatic leukemia and neutropenia secondary to chemotherapy, whose clinical picture is migraine, fever and rhynorrea. The images showed a compromise of the lateral wall of the sphenoidal cavity. He was treated with liposomal Amphotericin B, neutrophile growth factor and surgical resection in two opportunities with good clinical and radiological response.

Key words: Mucormycosis, liposomal Amphotericin B, neutrophile growth factor.

\footnotetext{
${ }^{1}$ Médico Otorrinolaringólogo, Unidad Docente Académica de Otorrinolaringología, Pontificia Universidad Católica de Chile.

${ }^{2}$ Interna Escuela de Medicina, Pontificia Universidad Católica de Chile.
} 


\section{INTRODUCCIÓN}

La mucormicosis es una afección causada por hongos saprófitos aeróbicos, que generalmente no son patógenos paraun huésped inmunocompetente, pero cuando se presenta en un paciente inmunocomprometido puede ser fatal y de rápida progresión. Es una enfermedad de baja prevalencia ${ }^{1}$. 1 desarrollo de ésta depende del estado inmune del huésped, por lo que generalmente estáasociadaa Diabetes Mellitus mal controlada, inmunosupresión ya sea mediadapor corticoides o por quimioterapia, y drogadicción endovenosa entre otros ${ }^{1,2}$. Los macrófagos y los neutrófilos son esenciales en la respuesta inmune contra estos microorganismos, previniendo la germinación de las esporas inhaladas².

日 sello de la mucormicosis es la invasión vascular por hifas que lleva a la trombosis arterial, infarto tisular, trombosis venosa, y hemorragia consecuente ${ }^{2}$.

La presentación clínica más frecuente es la forma rinocerebral, y el tratamiento recomendado incluye resección quirúrgica radical y uso de antimicóticos sistémicos como anfotericina $\mathrm{B}^{1}$.

Las alternativas terapéuticas se han expandido a otros fármacos entre los que se encuentran las nuevas formulaciones de anfotericina asociadas a lípidos, que intentan mejorar la solubilidad de las moléculas y así disminuir su toxicidad renal, manteniendo su eficacia clínica ${ }^{3}$. También se han desarrollado terapias de apoyo para mejorar la respuesta inmunológica de los huéspedes inmunosuprimidos, entre las que se encuentran los agentes estimuladores de colonias de neutrófilos que aumentan el conteo de éstos consiguiendo una más pronta restitución de la inmunidad celular, pero su real beneficio aún está en discusión.

Las ideas de este trabajo son revisar las terapias de apoyo que existen en el tratamiento de la mucormicosis, entre ellas:

1. Los factores estimulantes de células progenitoras de neutrófilos en pacientes neutropénicos

2. Las nuevas formulaciones de anfotericina, especialmente las de tipo liposomal.

3. Discutir cuál es el rol de la cirugía endoscópica en mucormicosis.

Con este fin, se presenta el caso de un paciente pediátrico con leucemia linfática aguda estirpe T y mucormicosis rinocerebral con compromiso de senos maxilar, esfenoidal y etmoidal izquierdos, fosa pterigomaxilar, rostrum del esfenoides y compromiso del sifón carotídeo que fue tratado con cirugía endoscópica y abierta, anfotericina B liposomal y un factor estimulador de colonias de neutrófilos (filgrastim), lográndose recuperación clínica y radiológica de la infección.

\section{CASO CLÍNICO}

Paciente de sexo masculino de 13 años con antecedentes de leucemia linfática aguda estirpe T, con 500 mil blastos al inicio de su enfermedad, de 4 meses de evolución, cursando su tercer ciclo de quimioterapia, hepatitis medicamentosa, neutropenia febril tratada, insuficiencia renal aguda que requirió diálisis, recuperada y síndrome de lisis tumoral.

Con historia de cefalea, dolor maxilar y rinorrea de 4 días de evolución, es evaluado por pediatra, quien realiza una radiografía de cavidades perinasales que evidencia velamiento del maxilar izquierdo.

Se inicia Ourocef ${ }^{\circledR}$; persistiendo con decaimiento, fiebre, náuseas y dejando de alimentarse. Se decide su hospitalización e interconsulta a otorrinolaringología Ingresa con neutropenia: 800 leucocitos $/ \mathrm{mm}^{3}$.

A examen físico se observan costras y rinorrea purulenta que ocupan la fosa nasal izquierda, en la TC de cavidades paranasales, se observa el compromiso de pared del seno esfenoidal (Figuras 1, 2 y 3 ).

Se realiza una cirugía endoscópica en la que se observa un cornete medio necrótico, se extirpa preservando sólo su raíz y se envía muestra a tinción de calcoflúor. Se reseca la pared medial del seno maxilar y se aspiran de éste abundantes secreciones, observándose una mucosa polipoidea que lo recubre.

Se resecan también la lámina perpendicular del etmoides y vómer, junto con celdillas de etmoides anterior y posterior. Además se realiza un abordaje de esfenoides con resección parcial de tabique interesfenoidal y extirpación de pólipos de aspecto vital.

日 informe de la tinción de calcoflúor señala que se visualizaron hifas con morfología propia de 

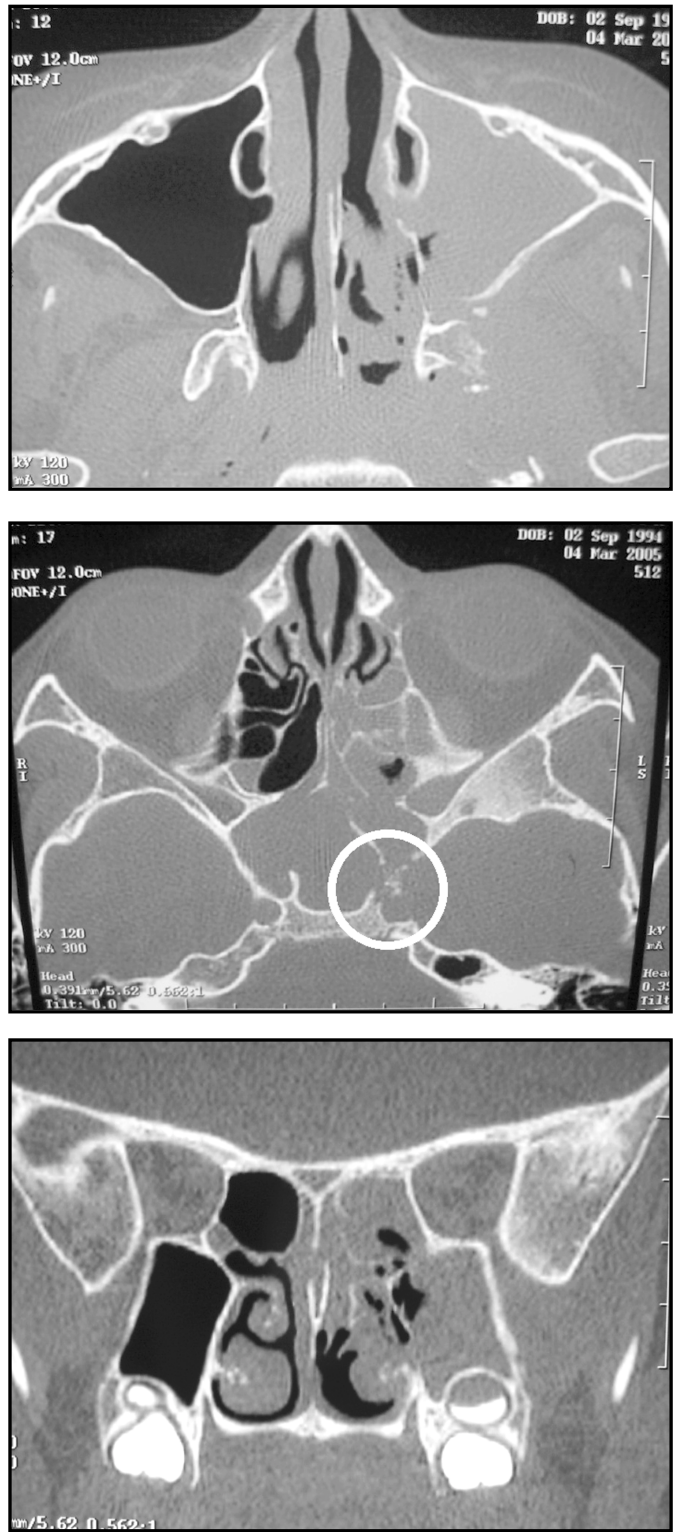

Figuras 1,2 y 3 . Rinosinusitis con componente osteolítico (círculos) en cortes axiales y coronales de la tomografía computada.

mucor; grandes, anchas, ramificadas, no septadas, en ángulo recto y la biopsia evidencia zygomicetes en los tejidos biopsiados que producen invasión vascular (Figura 4).

Presenta una evolución posquirúrgica favorable. Se inicia filgrastim (Neupogen ${ }^{\circledR}$ ), observándose una

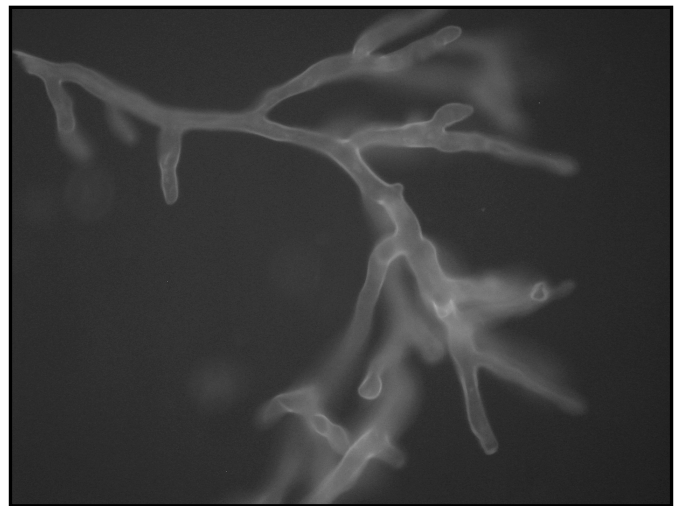

Figura 4. Tinción con calcoflúor de las hifas de zygomicetes.

elevación plasmática de $800 \mathrm{~mm}^{3}$ a $14.000 \mathrm{~mm}^{3}$ de leucocitos en 3 días.

Para conseguir niveles terapéuticos en forma precoz, se decide utilizar anfotericina liposomal con lo que se logra tratar al paciente con la mitad de la dosis total en 7 días (a $200 \mathrm{mg} /$ día por 5 días) y luego continuar con anfotericina convencional (1,5 $\mathrm{mg} / \mathrm{kg} /$ día hasta acumular dosis total de 2,5-3 gr).

En controles radiológicos posquirúrgicos a las 36 hrs de la cirugía, se observó que existía persistencia de focos osteolíticos en zonas contiguas a las resecadas quirúrgicamente (Figuras 5,6 y 7), incluyendo entre otros, la pared anterior del sifon carotídeo (Figura 8).

Dado la corta edad del paciente, y por lo tanto lo pequeño de sus cavidades paranasales, se realiza una segunda cirugía, con abordaje Caldwell-Luc combinado con cirugía endoscópica, en el que se observa una mucosa necrótica del seno maxilar debiendo abordar fosa pterigomaxilar; realizar re sección de lámina pterigoidea medial, parte de rostrum del esfenoides, pared lateral del seno esfenoidal, de pared anterior de sifón carotídeo, además de resecar de pólipos necróticos en esfenoides y parte del paladar óseo para poder remover la infección remanente.

日 paciente presenta una adecuada evolución, siendo controlado con curaciones frecuentes bisemanales durante los primeros 3 meses, existiendo ausencia de elementos sugerentes de infección tanto en el cuadro clínico como en la evalución radiológica (Figuras 9 y 10) . 


\section{DISCUSIÓN}

Las infecciones invasivas por hongos se han incrementado en las últimas décadas, debido al aumento en el número de pacientes en alto riesgo de contraerlas, que incluye a pacientes receptores de trasplante de órganos tanto sólidos como de medula ósea, neoplasias hematológicas y en tratamiento inmunosupresor.

Nuestro paciente se presentó con neutropenia secundaria a tratamiento con quimioterapia, el efecto adverso más frecuente en este tratamiento, lo que coloca a los pacientes en un grave riesgo de enfermedades fúngicas. Los hongos que con mayor frecuencia causan infecciones en los pacientes inmunosuprimidos son la Candida, el Aspergillus y el Mucor, como es este caso, en que se presentó una mucormicosis con compromiso de cavidades paranasales y del piso de fosa cerebral media.

Todos los agentes de la mucormicosis pertenecen al orden de los mucorales, tienen una amplia distribución, identificándose en varios sustratos, inclusive existen reportes de colonización de piel y mucosas en pacientes hospitalizados, sin tener éste un rol patogénico. Las colonias tienen un aspecto lanudo, a menudo parecen tenues puntos negros.

La mucormicosis es una enfermedad de baja frecuencia, se adquiere por la inhalación y coloniza ción de esporas en la vía aérea. Las hifas, a su vez, invaden los tejidos en especial la pared y lumen de los vasos sanguíneos provocando trombosis ${ }^{2}$.

日 término rinocerebral se emplea cuando se detecta compromiso de los senos paranasales, haya ocurrido o no invasión del sistema nervioso central .

Los síntomas iniciales, al igual que en nuestro pacienteson inespecíficos, lo que dificultael diagnóstico precoz Las formas más frecuentes de presenta ción son: dolor facial, rinorrea purulenta, epistaxis e hipoestesia nasal o facial. Se puede observar también cefalea y letargia en los casos avanzados, y son signos acompañantes la visión borrosa, diplopia, proptosis, amaurosis y alteración de conciencia?.

A examen físico el paladar y los cornetes pueden tener un aspecto gris o eritematoso lo que puede progresar hacia la formación de una masa necrótica o ulceración, aunque la mucosa sangra escasamente por la trombosis vascular.
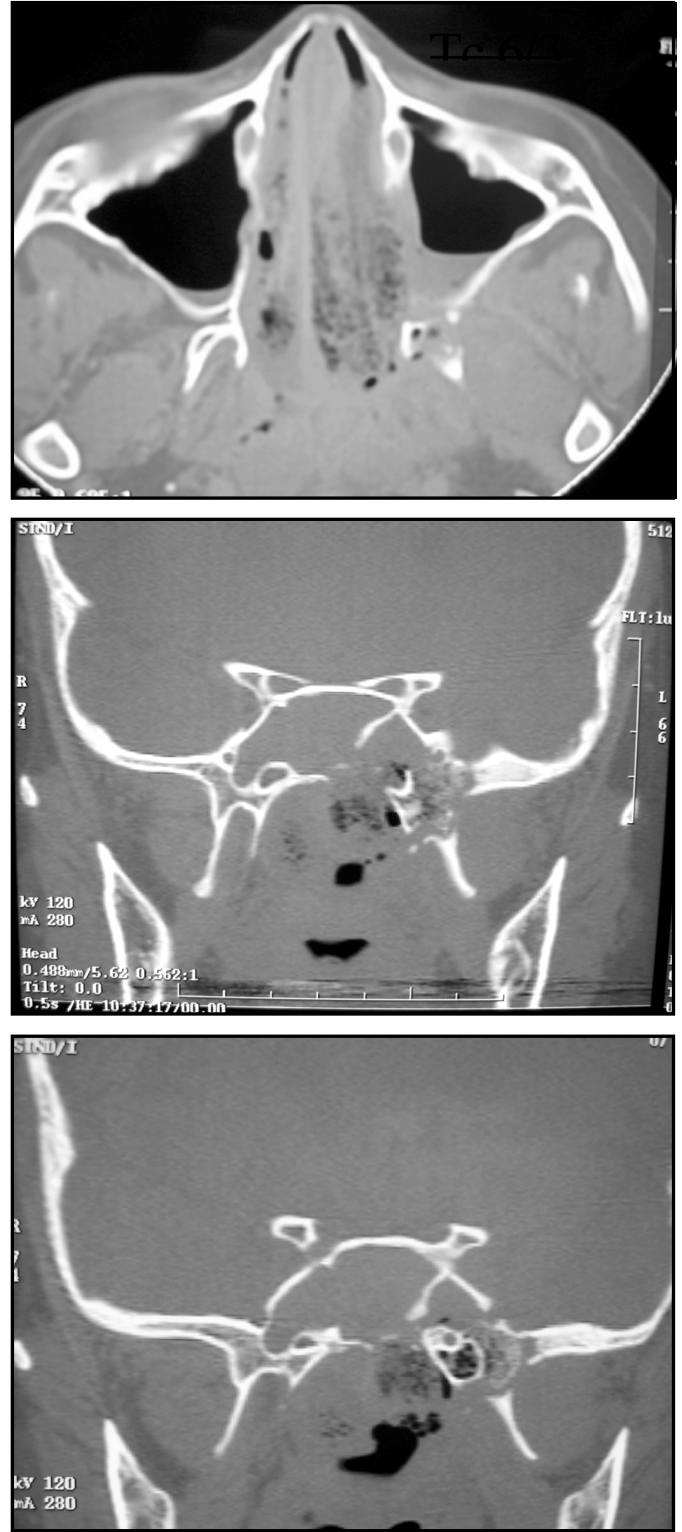

Figuras 5,6 y 7 . Persistencia de lesiones osteolíticas en pared lateral del seno y rostrum esfenoidales.

En la tomografía computada se puede observar el compromiso de las cavidades paranasales con destrucción ósea y en algunos casos se puede evidenciar invasión de la órbita, trombosis del seno cavernoso y edema cerebral. La resonancia muestra estas alteraciones en forma más precoz teniendo mayor sensibilidad para definir el compromiso vascular ${ }^{2}$. 


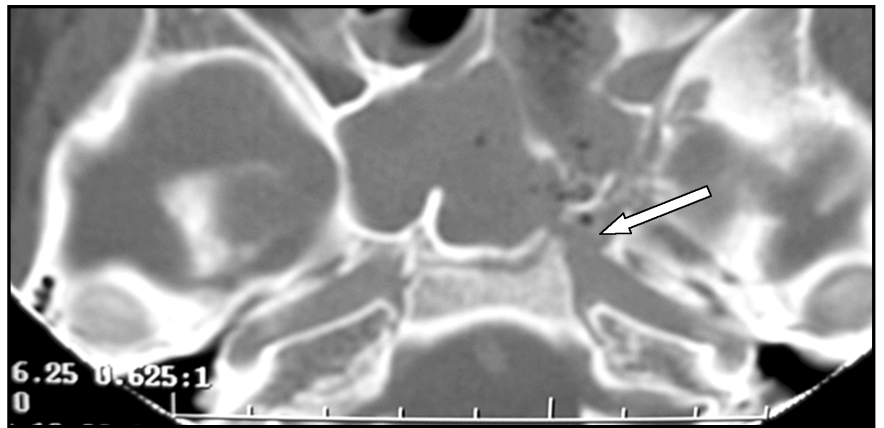

Figura 8. Compromiso de pared anterior de sifón carotídeo en corte axial (tomografía computada).
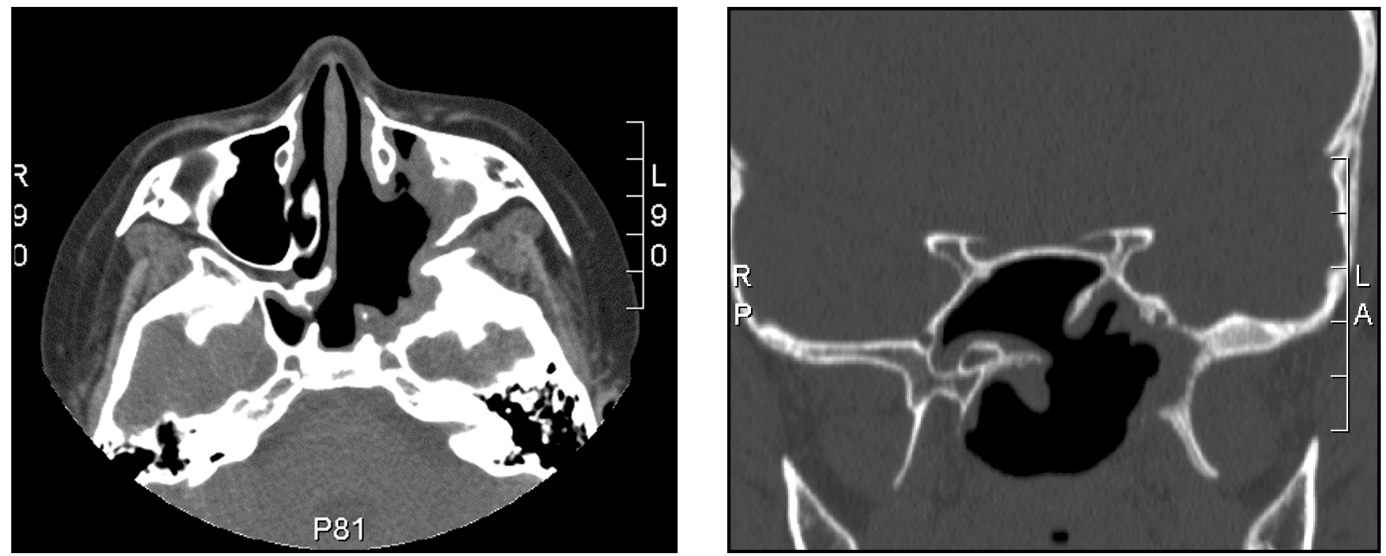

Figuras 9 y 10. Control topográfico a los 3 meses de iniciado el tratamiento.

日 tratamiento se basa en 3 pilares fundamentales: tratamiento de la enfermedad de base, terapia antifúngica sistémica y cirugía.

La anfotericina B es actualmente el agente de elección en la mayoría de las infecciones por hongos, por su efecto fungicida de amplio espectro con un mínimo riesgo de desarrollar resistencia, pero está asociada a una toxicidad significativa, tanto aguda como crónica, lo que limita su uso. Por ello, se han desarrollado otros antifúngicos sistémicos para aumentar su tolerancia Se han aprobado tres formulaciones lipídicas para el tratamiento de infecciones invasivas por hongos en pacientes no tolerantes o resistentes a la anfotericina convencional: anfotericina con complejo lipídico, anfotericina en dispersión coloidal, y la anfotericina B liposomal o ambisome; los tres representan un método mejorado de entrega de la droga; difieren tanto en composición como en farmacocinética 5 . No está claro aún si estas diferencias son importantes en cuanto a su tolerancia y eficacia, pero existen estudios que sugieren que la anfotericina liposomal es la menos nefrotóxica de las formulaciones lipídicas $(14,6 \%)$, mientras que la anfotericina convencional lo sería mucho más $(33,2 \%)^{6,7}$.

Para nuestro paciente se dispuso del uso de anfotericina B liposomal, en la que se encapsula la anfotericina en liposomas, lo que reduce su toxicidad y permite aumentar las dosis. $\mathrm{\theta}$ ambisome es un liposoma esférico, pequeño, unilamelar, con un tamaño promedio de $80 \mathrm{~nm}^{8,9}$ y permanece como una esfera intacta y estable por períodos prolongados dentro del organismo, lo que le otorga propiedades farmacocinéticas diferentes a las otras formulaciones lípidicas, entre las que se encuentra menor clearancey mayor duración de la concentra- 
ción plasmática óptima9 ${ }^{9}$ 日 liposoma se acumularía en el sitio de infección y su disrupción ocurriría luego de estar adherido a la pared celular del hongo, lo que resultaría en la unión de la anfotericina $\mathrm{B}$ con el ergosterol de la membrana celular y en la subsecuente lisis celular.

Tiene la ventaja de preservar mejor la indemnidad de las membranas citoplasmáticas del huésped, de manera que aun con dosis elevadas del fármaco, el tratamiento es bien tolerado, con menor nefrotoxicidad y tendría una mayor actividad antifúngica en el sitio de la infección al permitir comenzar el tratamiento con dosis de carga mucho mayores ${ }^{10}$.

Es aun motivo de controversia la dosis requerida, generalmente se utiliza 1-4 mg/kg/día. Estudios sugieren que dosis bajas serían igual de eficaces ${ }^{11}$. Los efectos adversos son infrecuentes, siendo la hipokalemia el más común (18\%) ${ }^{12}$.

La neutropenia es un efecto adverso de la quimioterapia frecuente y peligroso, que muchas veces obliga a una disminución de la dosis o suspensión de ésta.

En el caso de nuestro paciente, observamos que el uso de factores estimulantes de colonias de neutrófilos (Neupogen o filgrastim) permitió un rápido aumento en el conteo de éstos, consiguiendo una más pronta restitución de la inmunidad celular. 日 Neupogen ${ }^{\circledR}$ es un factor estimulante de colonias de granulocitos (G-CSF) producido por tecnología DNA-recombinante. Es una proteína de 175 aminoácidos producida por la bacteria Escherichia coli dentro de la cual se ha insertado el gen del factor estimulante de granulocitos ${ }^{4}$. En cuanto al mecanismo de acción, se une en forma específica a receptores de superficie de las células hematopoyéticas y estimula la proliferación, diferenciación y activación funcional selectiva de varias células finales ${ }^{4}$. Su dosis por vía subcutánea o intravenosa $5 \mathrm{mg} / \mathrm{kg} /$ día en una sola inyección diaria durante dos semanas hasta alcanzar un recuento de leucocitos de $10.000 / \mathrm{mm}^{3}$. Se ha visto que el Neupogen ${ }^{\circledR}$ reduce tanto la intensidad como la duración de la neutropenia consecutiva a la quimioterapia y disminuye la incidencia, duración y gravedad de las infecciones que acompañan a la neutropenia en estos pacientes; permitiendo de esta manera que los pacientes puedan completar sus ciclos de quimioterapia ${ }^{13}$. No se ha observado una mejoría en la sobrevida pero disminuiría el número de hospitalizaciones, uso de antibióticos endovenosos, y neutropenias febriles con una mejoría en la calidad de vida14-17. Sin embargo, ello no ha sido confirmado en otros estudios, donde no se demostró una disminución en la incidencia de infecciones, ni en el número de hospitalizaciones ${ }^{15}$. No se han descrito efectos secundarios graves, pero se ha visto dolor músculo-esquelético, hipotensión, fiebre, diarrea y fatiga entre otros. Actualmente su indicación incluye el tratamiento de pacientes con varias condiciones neutropénicas como: secundaria a quimioterapia, trasplante autólogo de médula ósea, neutropenias congénitas crónicas, idiopáticas, cíclicas de niños, producidas por radioterapia, esteroides, antiinflamatorios, AZT, y drogas en general y en pacientes con infección y leucopenia; pero su gran costo limita su uso masivo ${ }^{18-19}$.

A través de la conjugación del filgrastim con un polietilenglicol (pegfilgrastim); se protege a las droga del clearance renal, de su degradación enzimática y del reconocimiento por la inmunidad celular; logrando aumentar su vida media, administrándola una vez en cada ciclo de quimioterapia sin tener la necesidad de repetir dosis diarias, con igual recuperación del número absoluto de neutrófilos, con una elevación más sostenida en el tiempo a diferencia de los peaks que se producen con el filgrastim y con un menor número de neutropenias febriles. Pegfilgrastim estimula la proliferación y diferenciación de neutrófilos a través del mismo mecanismo que filgrastim ${ }^{20}$.

Existen publicaciones que han descrito otras terapias de apoyo con algún grado de utilidad, como el uso de irrigaciones locales de anfotericina B y agua oxigenada; como también el uso de oxigeno hiperbárico $22-24$.

La cirugía endoscópica es una excelente terapia en casos seleccionados de mucormicosis inicial. Puede combinarse con cirugía externa otorgando una excelente visión de las lesiones provocadas por mucor. En el abordaje de fosa pterigomaxilar, puede complementar la visión a través del Caldwell Luc, especialmente en nuestro caso en que las cavidades paranasales eran muy pequeñas por la temprana edad del paciente. 


\section{BIBLIOGRAFIA}

1. MM Pía SPaLONI M, P Gaser $K, P$ Verduco $L$. Mucormicosis Rinocerebral: Sobrevida en un niño con leucemia Rev Chil Infect 2004; 21: 53-56.

2. WM Pía SPALloni M, A OHÁvez P, Cl AVILES L, J Corfé G Mucormicosis en Pediatría. Rev Chil Infect 2004; 21: 17-25.

3. A FCA C. Tratamiento de infecciones fúngicas sistémicas. II parte: Anfotericina $B$, aspectos farmacoeconómicos y decisiones terapéuticas. Rev Chil Infect 2004; 21: 317-326.

4. Neupogen - Roche. Ourr Hematol Rep. 2002; 1(2): 95-102.

5. HANN IM, PRENTICE HG Lipid-based amphotericin B: a review of the last 10 yearsofuse. J Cin Pharm Ther. 1999; 24(4): 249-57.

6. Gros SB, Ohapuis F, Dequliter E, Reva BG Adverse effects of antifungal therapies in invasive fungal infections: review and metaranalysis. Eur $J$ Cin Microbiol Infect Dis. 2005; 24(2): 119-30.

7. Badia X, Roset M, Carreras E, Ausin I, Herre RA L. Meta-analysis on safety and efficacy of liposomal amphotericin Bin the empirical treatment of febrile neutropenia Med Cin(Barc), 2004; 122(16): 610-6.

8. TOLlgMAR J, KLINGSPOR L, RINGDEN O. Liposomal amphotericin B (AmBisome) for fungal infections in immunocompromised adults and children. Cin Microbiol Infect. 2001; 7 Suppl 2: 68-79.

9. Boswel GW, BUEL D, BeKERSKY I. AmBisome (liposomal amphotericin B): a comparative re view. An Atorrinolaringol Ibero Am. 1998; 25(1): 45-56.

10. RITTER J. Amphotericin B and its lipid formulations. Mycoses. 2002; 45 Suppl 3: 34-8.

11. ELIS M. Amphotericin B preparations: a maximum tolerated dose in severe invasive fungal infections? Transpl Infect Dis. 2000; 2 (2): 51-61.

12. HAY RJ. Liposomal amphotericin B, AmBisome. J Infect. 1994; 28 Suppl 1: 35-43.

13. DALE DC. Colony-stimulating factors for the management of neutropenia in cancer patients. Cancer Treat Rev. 2002; 28 Suppl A: 7-11.

14. VALLEY AW. New treatment options for managing chemotherapy-induced neutropenia Am J Health Syst Pharm. 2002; 59 (15 Suppl 4): S11-7.

15. HoLDSWORTH MT, MATHEW P. Efficacy of colonystimulating factors in acute leukemia. Ann Pharmacother. 2001; 35 (1): 92-108.

16. SYLVESTER RK. Cinical applications of colonystimulating factors: a historical perspective. Am J Health Syst Pharm. 2002; 59 (7 Suppl 2): S6-12.

17. FRAMPTON JE LE CR, FAULDS D. FLGRASTIM. A review of its pharmacological properties and therapeutic efficacy in neutropenia. Drugs. 1994; 48(5): 731-60.

18. LYMAN GH, KUDERER NM. Flgrastim in patients with neutropenia: potential effects on quality of life. Drugs. 2002; 62 Suppl 1: 65-78.

19. DALE DC. Colony-stimulating factors for the management of neutropenia in cancer patients. Drugs. 2002; 62 Suppl 1: 1-15.

20. CRAWFORD J. Pegfilgrastim administered once per cycle reduces incidence of chemotherapyinduced neutropenia Drugs. 2002; 62 Suppl 1:65-78.

21. DanNaOUI E, MeEtiadIS J, MOUTON JW, MeS JF, VघRW曰J PE Eurofung Network.In vitro susceptibilities of zygomycetes to conventional and new antifungals. $J$ Antimicrob Chemother. 2003; 51(1): 45-52.

22. Seff SR, GHoO PH, CaRter SR. Role of local amphotericin B therapy for sino-orbital fungal infections. Ophthal Plast Reconstr Surg. 1999; 15(1): 28-31.

23. Bentur $Y$, Shupak A, Ramon Y, Abramovich A, WolfN G STEN H, KRIVO N. Hyperbaric oxygen therapy for cutaneous/soft-tissue zygomycosis complicating diabetes mellitus. Rev Stomatol Chir Maxillofac. 1998; 99(1): 52-5.

24. Blaine DA, Frable MA Mucormycosis. Adjunctive therapy with hydrogen peroxide. Semin Oncol. 1990; 17 (3 Suppl 6): 6-9.

\footnotetext{
Dirección: Dr Rodrigo Iñiguez C.

Hospital Cínico de la P Universidad Católica de Chile.

Av. Ricardo Lyon 249, dpto. 32. Providencia. Santiago, Chile. Email: riniguez@uc.cl
} 